\title{
In Silico Docking for Inhibition Neuropilin-1 (SARS- CoV-2 receptor) by Some Natural and Approved Drugs
}

mohamed seadawy ( $\sim$ biologist202054@yahoo.com )

Main chemical laboratories, Egypt Army

Mohamed Shamel

MCL

Aya Ahmed

$\mathrm{NCl}$

Abdel Rahman N. Zekri

$\mathrm{NCl}$

\section{Research}

Keywords: SARS-CoV-2, Spike (S) protein, Neuropilin-1

Posted Date: December 3rd, 2020

DOl: https://doi.org/10.21203/rs.3.rs-118263/v1

License: (c) (i) This work is licensed under a Creative Commons Attribution 4.0 International License.

Read Full License 


\section{Abstract}

Background: Neuropilin-1 (NRP-1) is a multifunctional transmembrane receptor for ligands that affect developmental axonal growth and angiogenesis. Beside its role in cancer, NRP-1 is a reported entrance for several viruses, including severe acute respiratory syndrome coronavirus 2 (SARS-CoV-2), the causal agent of coronavirus disease 2019 (COVID-19).

Methods: We made Insilco docking between the spike protein and Neuropilin-1 using Cluspro 2.0 software. Therefore, Neuropilin-1 becomes host factor for SARS-CoV-2 infection. Then by using molecular docking, we test nine compounds against Neuropilin-1 for its inhibition.

Results: Our result revealed that NRP-1 receptor is considered as Therapeutic target for SARS-CoV2 treatment and screened with natural compounds and drugs (e.g. Carvacrol, Thymol, Amantadine, Daclatasvir, Ravidasvir, Remdesivir, Sofosbuvir, Hesperidine and Thymoquinone) by molecular docking study.

Conclusion: These natural products and drugs may emerge as potential Neuropilin-1 inhibitor. However, additional exploration is predictable for the investigation of the essential use of the drugs and herbs containing these natural products and their in-vivo activity.

\section{Background}

NEUROPILIN-1 (NRP-1) is the receptor involved in the development of the cardiovascular system. It mediates the chemorepulsant activity of semaphorins. It involved in the formation of certain neuronal circuits. It also binds to semaphorin 3A. It Regulates mitochondrial Iron.SARS-CoV-2 attachment with host cell receptor is catalyzed by Furin, which cleaves the Spike (S) protein at a specific sequence motif that generates a polybasic (Arg-Arg-Ala-Arg) C-terminal sequence on S1. The sequence motif conforms to the $\mathrm{C}$-end rule, which means that the C-terminal sequence may allow the protein to bind with cell receptor neuropilin-1 and neuropilin-2 receptors (1).

\section{Material And Methods}

\section{Protein molecular modeling of spike protein fragment and NRP-1 protein:}

Swiss Model web server was utilized to build the 3D structural model for Egyptian sequences for NRP-1 and the spike protein (2). The two solved structures with PDB ID: 7QQm and 6XR8 were chosen to be the homolog solved structures for NRP-1 and the spike protein since they share a sequence identity of $100 \%$ and $99.84 \%$, respectively $(3,4)$. After model building, the models were validated through the Structural Analysis and Verification Server (SAVES) webserver of the UCLA and Molprobity from Duke University (5).

Molecular docking between S protein fragment and NRP-1. 
Molecular docking studies between S protein fragment and human NRP-1 receptor are performed using ClusPro (6). Following equation has been used to compute cluster scores as well as to predict the lowest binding energy (using ClusPro 2.2 online server (7)

$\mathrm{E}=0.40 \mathrm{E}_{\mathrm{rep}}+-0.40 \mathrm{E}_{\mathrm{att}}+600 \mathrm{E}_{\text {elec }}+1.00 \mathrm{E}_{\mathrm{DARS}}$.

The repulsive (rep), attractive (att), electrostatic (elec) forces and interactions extracted from the decoys as the reference state (DARS), are measured using molecular docking study (7).

\section{Molecular docking study of some natural compounds and FDA approved drugs}

The tested compounds are retrieved from the PubChem database and prepared using PyMOL software (8, 9). Docking experiments were performed using AutoDock Vina software (10). Model built for NRP-1 was used in this study, and its binding affinities against, Carvacrol, Thymol, Amantadine, Daclatasvir, Ravidasvir, Remdesivir, Sofosbuvir, Hesperidine, and Thymoquinone were tested using the Vina scoring function. Chimera software was used to represent and analyze the docking complexes.

\section{Results}

Molecular docking between spike protein fragment and human NRP-1 receptor.

Human NRP-1 receptor (PDB ID 2QQM) is considered as receptor protein for molecular docking study of spike protein fragment with its receptor in human host. By using ClusPro 27 web server, docking structure of A chain of human NRP-1 receptor binds with SARS CoV2 spike protein fragment with binding energy $-1219.1 \mathrm{kcal} / \mathrm{mole}$.

\section{Hesperidine, Ravidasvir, Daclatasvir, Remdesivir and sofosbuvir for inhibition of NRP-1}

There are 9 natural and FDA approved drugs tested against NRP-1 human receptor for its inhibition (table 1). Hesperidin binds with NRP-1 protein fragment with binding energy $-9 \mathrm{kcal} / \mathrm{mole}$. This docked structure is stabilized by four $\mathrm{H}$ binding (shown in Figure 3 with orange lines) at SER 24 of NRP-1 with 0 atom of hesperidin, with bond length $2.403^{\circ} \mathrm{A}$ and three $\mathrm{H}$ binding at GLY 5 with $\mathrm{O}$ atom of hesperidin with a distance $2.305,2.2$ and $2.803^{\circ} \mathrm{A}$.

Ravidasvir binds with human receptor NRP-1 protein, at GLY 9. But with higher binding energy -10.3 $\mathrm{kcal} / \mathrm{mole}$ compared to that of hesperidin $-9 \mathrm{kcal} / \mathrm{mole}$, with bond length $2.198^{\circ} \mathrm{A}$.

Daclatasvir, Remdesivir and Sofosbuvir bind with human receptor NRP-1 protein, at TYR 28, HIS 3 and GLY 37 respectively. However, with less binding energy $-8,-7.6,-7.3 \mathrm{kcal} / \mathrm{mole}$ respectively compared to that of hesperidin and Ravidasvir, with bond length $1.828,2.037$ and $2.316^{\circ} \mathrm{A}$ as shown in figure 2 and 3 . 
Table 2. The binding affinity (in kcal/mole) of tested compounds against the NRP-1 as a SARS-CoV2 target calculated using AutoDock Vina software

\begin{tabular}{|ll|}
\hline Ligand & Affinity $(\mathrm{kcal} / \mathrm{mole})$ \\
\hline Amantadine & -5.6 \\
\hline Carvacrol & -5.4 \\
\hline Daclatasvir & -8.0 \\
\hline Hesperidine & -9.0 \\
\hline Ravidasvir & -10.3 \\
\hline Remdesivir & -7.6 \\
\hline Sofosbuvir & -7.4 \\
\hline Thymol & -5.3 \\
\hline Thymoquinone & -5.4 \\
\hline
\end{tabular}

\section{Discussion}

As reflected from our results Hesperidine, Ravidasvir, Daclatasvir, Remdesivir and Sofosbuvir show perfect average binding affinities against Neuropilin-1 model from the Egyptian sequence.

Considering the primary sequence of Neuropilin-1, a homology modelled structure is built using SWISSMODEL, with template (PDB ID 2QQM) having sequence identity $100.00 \%$ and coverage $100 \%$. This stable human host Neuropilin-1 receptor protein is used for binding with spike protein fragment by molecular docking study.

Binding affinity of spike protein fragment with its Neuropilin-1 receptor is very high with binding energy $-1219.1 \mathrm{kcal} / \mathrm{mole}$.

Structure of Neuropilin-1 receptor protein is considered as therapeutic target for SARS-CoV2 treatment and screened with natural compounds and drugs e.g. Carvacrol, Thymol, Amantadine, Daclatasvir, Ravidasvir, Remdesivir, Sofosbuvir, Hesperidine, and Thymoquinone by molecular docking study. Among them, hesperidin binds with SER 24 with $\mathrm{O}$ atom of hesperidin, with bond length $2.403^{\circ} \mathrm{A}$ and three $\mathrm{H}$ binding at GLY 5 with $\mathrm{O}$ atom of hesperidin with a distance $2.305,2.2$ and $2.803^{\circ} \mathrm{A}$ with human NRP-1 receptor protein. Similarly, Ravidasvir binding amino acid i.e. GLY 9 is present on NRP-1 molecule.

Hesperidin is a major flavonoid compound, present in orange and lemon fruits. $470-761 \mathrm{mg} / \mathrm{L}$ of Hesperidin is normally present in orange juice (11). This phytochemical exhibit various medicinal 
properties. According to oral toxicity study of hesperidin, it can be concluded that this phytochemical can be safely used in herbal formulations with its $L D_{50}$ value more than $2000 \mathrm{mg} / \mathrm{kg}(11)$.

Ravidasvir (RDV) is a new group pan genotypic hepatitis C virus (HCV) NS5A inhibitor, with high barrier to baseline resistance-associated species (12).

\section{Conclusion}

Since, most of the drug candidates presently available for COVID-19 significantly act on viral spike protein and other studies reports that NRP-1 is an entrance for several viruses, including severe acute respiratory syndrome coronavirus 2 (SARS-CoV-2). By using molecular docking analysis, we have predicted the inhibitor activity of several natural products that can emerge as potential drug candidates inhibiting the binding between virus spike protein and NRP-1. A promising binding of natural products and drugs with the NRP-1 was revealed by docking analysis. Among the several natural products and drugs screened by docking analysis, Hesperidine, Ravidasvir, Daclatasvir, Remdesivir and Sofosbuvir were found to exhibit a higher degree of interaction with the human receptor accompanied by lowest binding energy with favorable drug like properties. Thus, these natural products and drugs may emerge as potential Neuropilin-1 inhibitor. However, additional exploration is predictable for the investigation of the essential use of the drugs and herbs containing these natural products and their in-vivo activity.

\section{Abbreviations}

Neuropilin-1: NRP-1

Coronavirus Disease 2019: COVID-19

Severe acute respiratory syndrome coronavirus 2: SARS-CoV-2

Spike Protein: S Protein

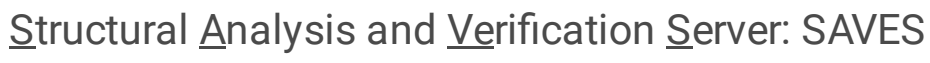

Repulsive: rep

Attractive: att

Electrostatic: elec

Decoys as the reference state: DARS

\section{Declaration}

-Ethics approval and consent to participate 
Not applicable

-Consent to publication

Not applicable

- Availability of data and material

The docking structures are available upon request from the corresponding author.

- Competing interests

All the authors declare that there is no competing interest in this work.

- Funding

Not applicable

- Authors' contributions

1- Mohamed Gomaa Seadawy

Writing, Editing and Molecular Docking

2- Mohamed Shamel Eldesoky

Data analysis

3- Aya Ahmed Saeed Mohamed

Molecular docking and data analysis

4- Abdel Rahman Nabwi Zekri

Revising

- Acknowledgements

Not applicable

\section{References}

1. Hoffmann, M. et al. A multibasic cleavage site in the spike protein of SARS-CoV-2 is essential for infection of human lung cells. Mol Cell 78, 779-784 (2020).

2. Biasini M, Bienert S, Waterhouse A, Arnold K, Studer G, Schmidt T, et al. SWISS-MODEL: modelling protein tertiary and quaternary structure using evolutionary information. Nucleic Acids Research. 
2014;42:W252-W8.

3. Chi X, Yan R, Zhang J, Zhang G, Zhang Y, Hao M, et al. A neutralizing human antibody binds to the Nterminal domain of the Spike protein of SARS-CoV-2. Science (New York, NY). 2020;369:650-5.

4. Wang Q, Wu J, Wang H, Gao Y, Liu Q, Mu A, et al. Structural Basis for RNA Replication by the SARSCoV-2 Polymerase. Cell. 2020;182:417-28.e13.

5. Williams CJ, Headd JJ, Moriarty NW, Prisant MG, Videau LL, Deis LN, et al. MolProbity: More and better reference data for improved all-atom structure validation. Protein Science. 2018;27:293-315.

6. Kozakov, D. et al. The ClusPro web server for protein-protein docking. Protoc. 12(2), 255-278 (2017).

7. Kozakov, D. et al. How good is automated protein docking? Struct. Funct. Bioinf. 81(12), 2159-2166 (2013).

8. 7.6 V. The PyMOL Molecular Graphics System, Version 1.7.6 Schrödinger, LLC.

9. Kim S, Thiessen PA, Bolton EE, et al. PubChem substance and compound databases. Nucleic acids research 2015; 44(D1): D1202-D13.

10. Trott O, Olson AJ. AutoDock Vina: improving the speed and accuracy of docking with a new scoring function, efficient optimization, and multithreading. J Comput Chem. 2010;31:455-61.

11. Rakesh, S., Chapter 59-polyphenols in health and disease: Practice and mechanisms of benefits. In Polyphenols in Human Health and Disease(eds. Ronald, R. W., Victor, R. P., Sherma, Z.), 757-778 (Academic Press, 2014).

12. Xu, X., Feng, B., Guan, Y., Zheng, S., Sheng, J., Yang, X., Ma, Y., Huang, Y., Kang, Y., Wen, X. and Li, J., 2019. Efficacy and Safety of All-oral, 12-week Ravidasvir Plus Ritonavir-boosted Danoprevir and Ribavirin in Treatment-naïve Noncirrhotic HCV Genotype 1 Patients: Results from a Phase 2/3 Clinical Trial in China. Journal of clinical and translational hepatology, 7(3), p.213.

\section{Figures}


A

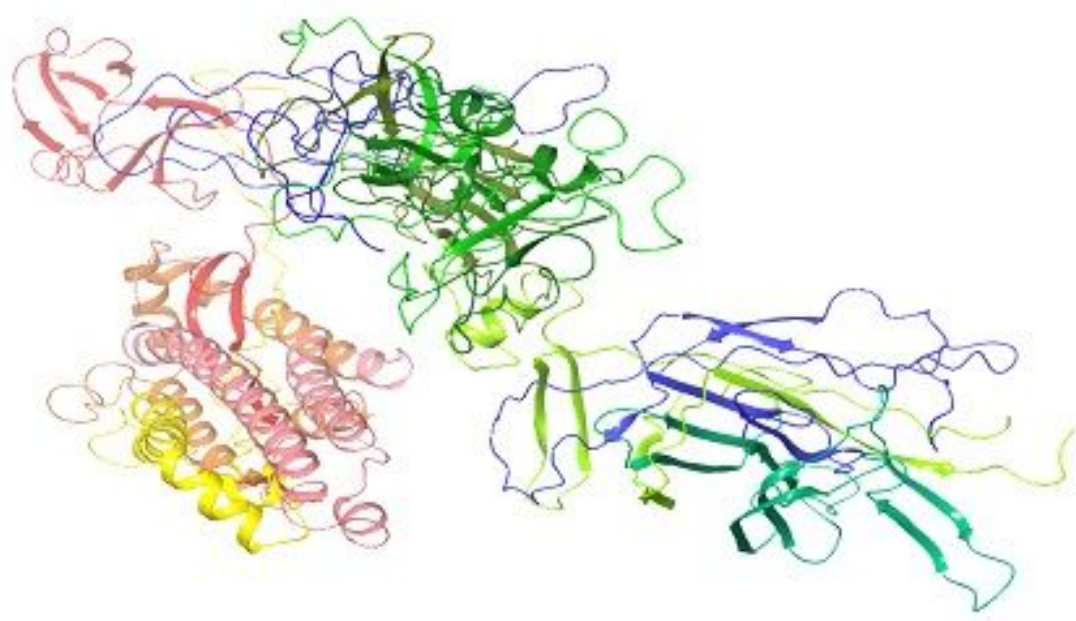

B

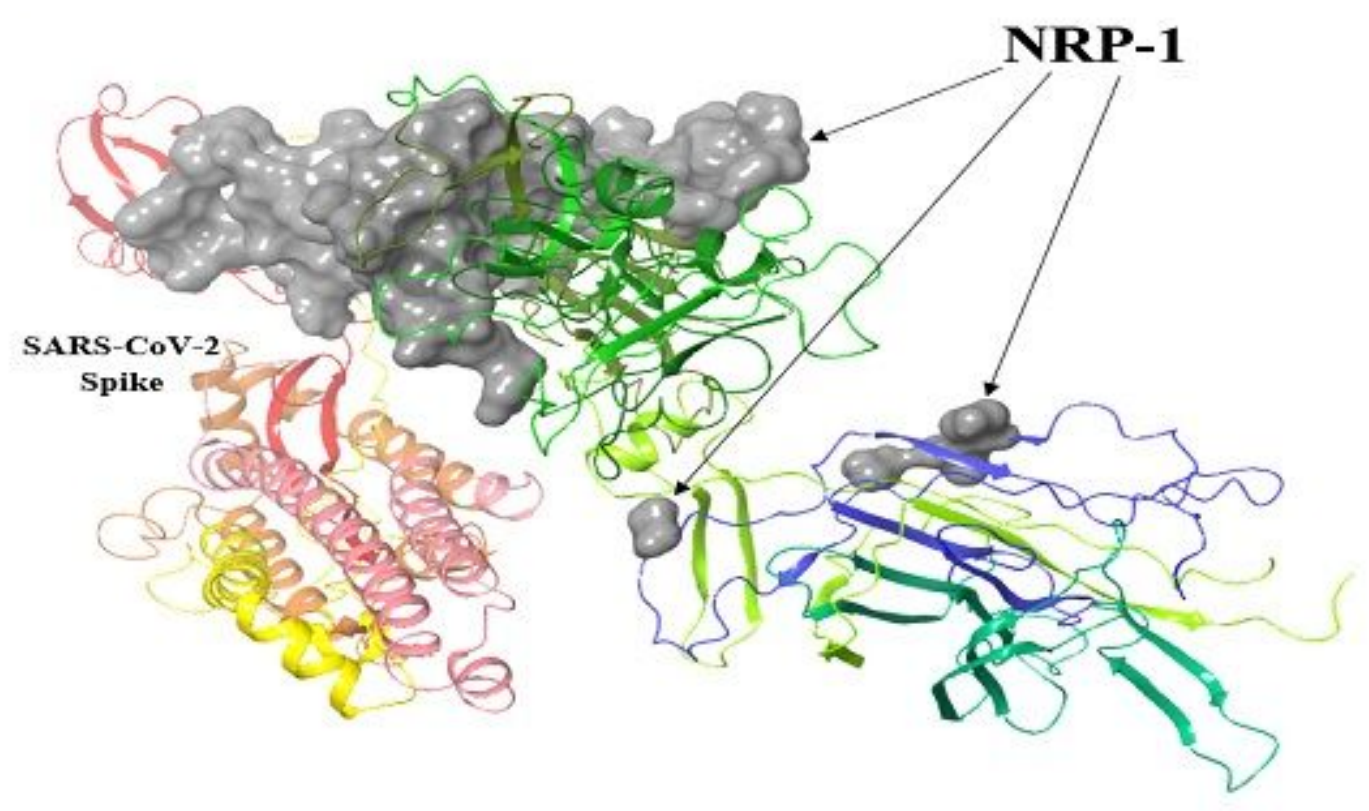

Figure 1

A and B The 3D structures of the Spike protein of SARS-CoV-2 docked with Neuropilin-1 human receptor. 
A

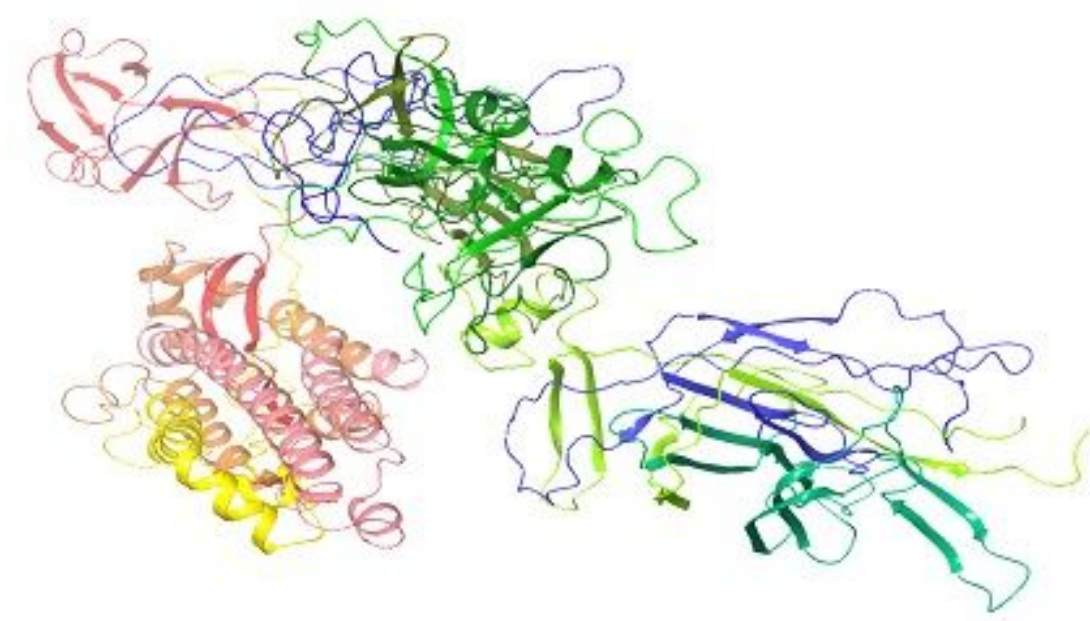

B

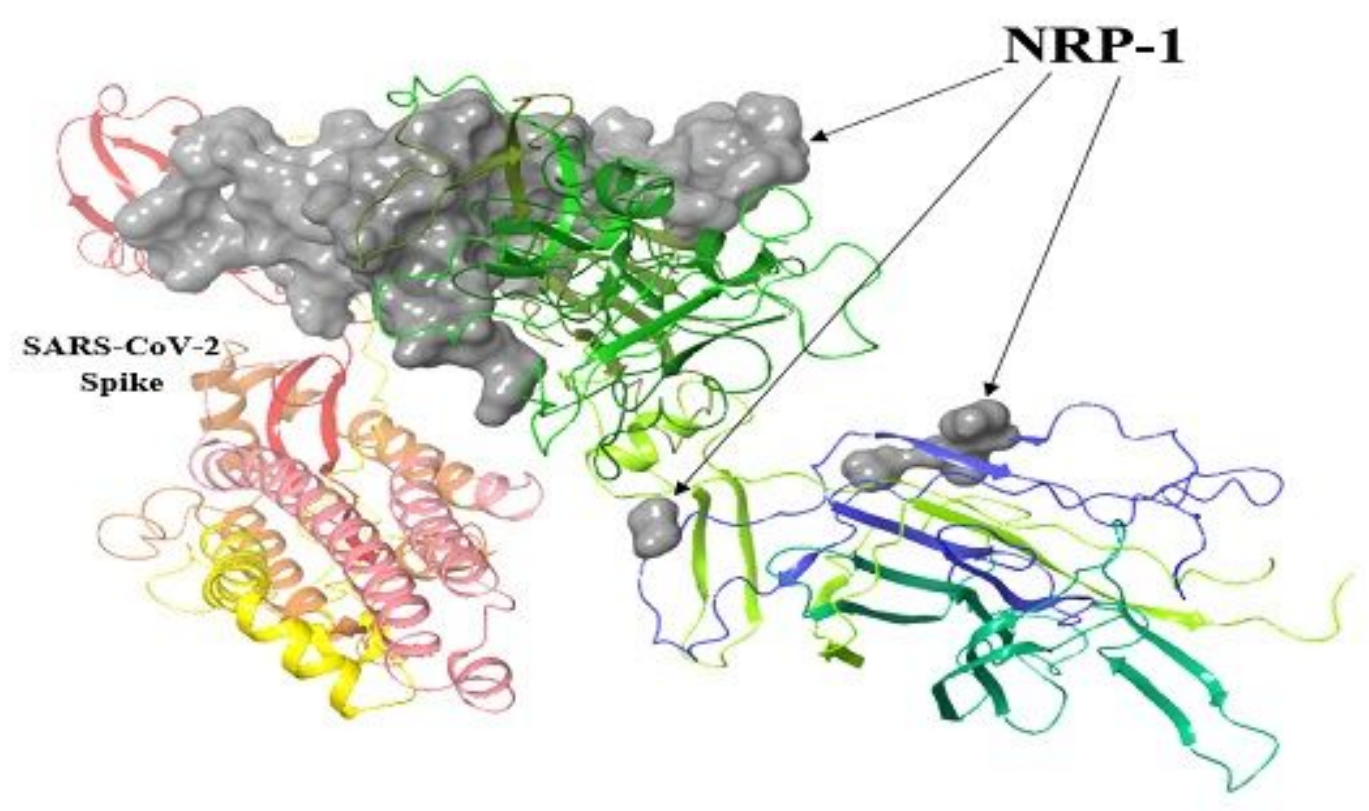

Figure 1

A and B The 3D structures of the Spike protein of SARS-CoV-2 docked with Neuropilin-1 human receptor. 
A
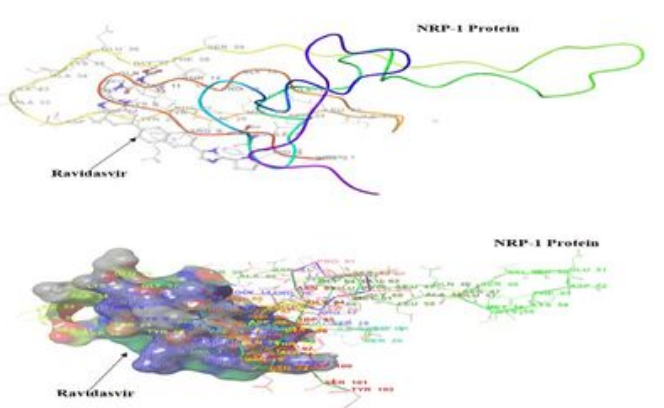

B
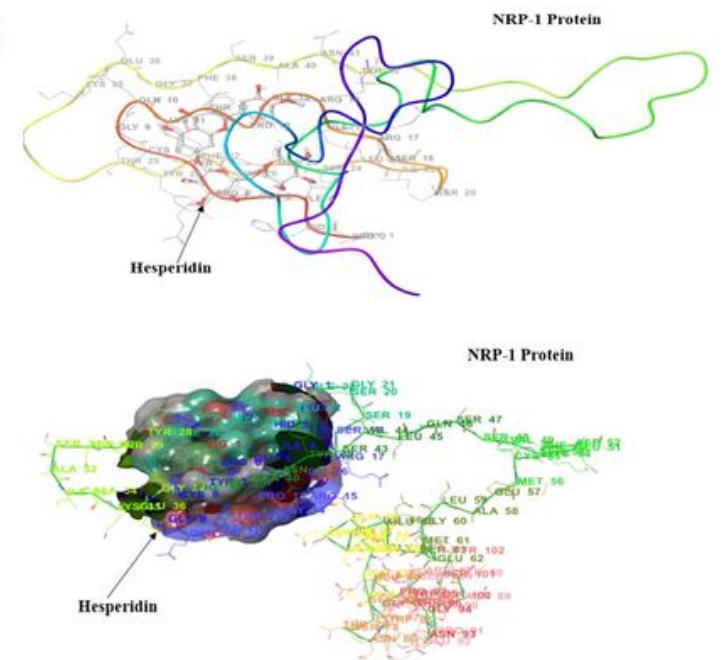

C
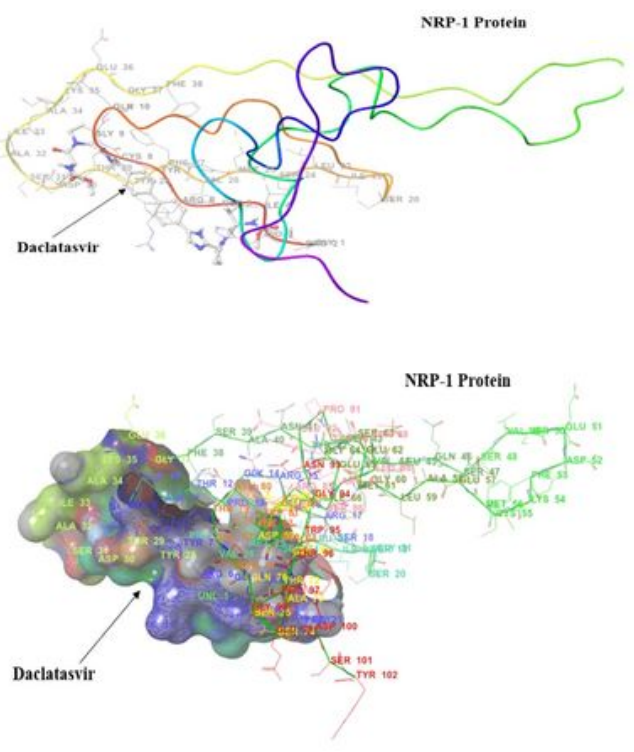

D
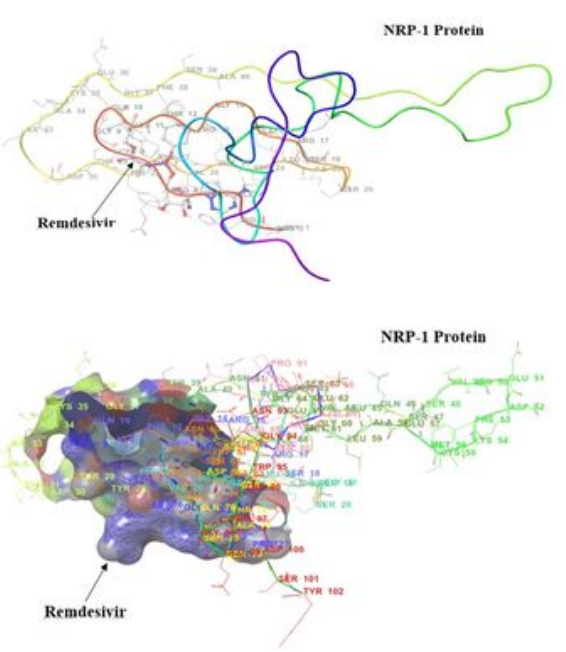

E
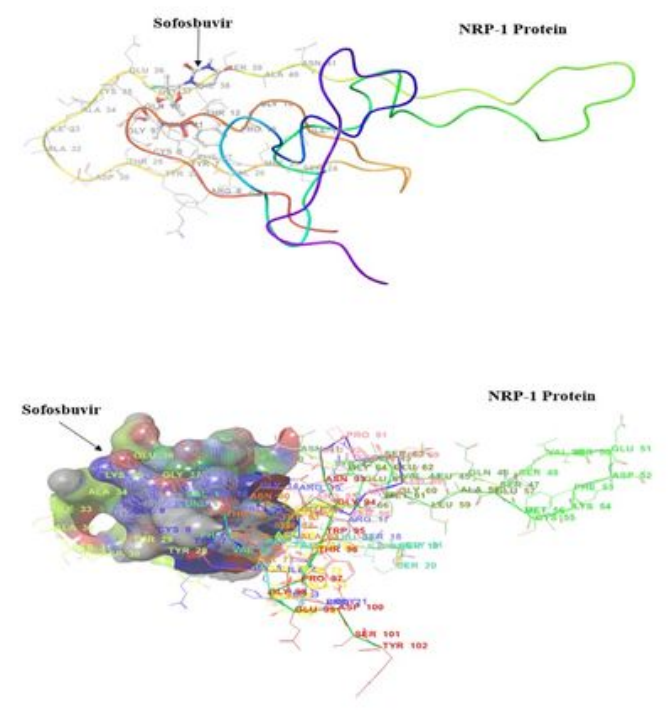

\section{Figure 2}

The docking complexes formed after the docking of (A) Ravidasvir, (B)Hesperidin, (C) Daclatasvir, (D) Remdesivir, and (E) Sofosbuvir into the Neuropilin-1 human receptor active site. 
A
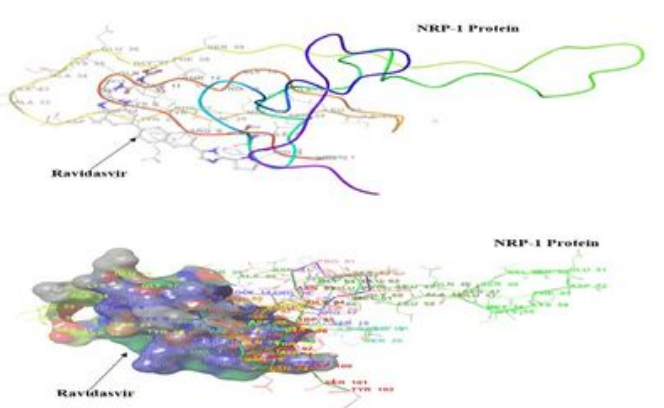

B
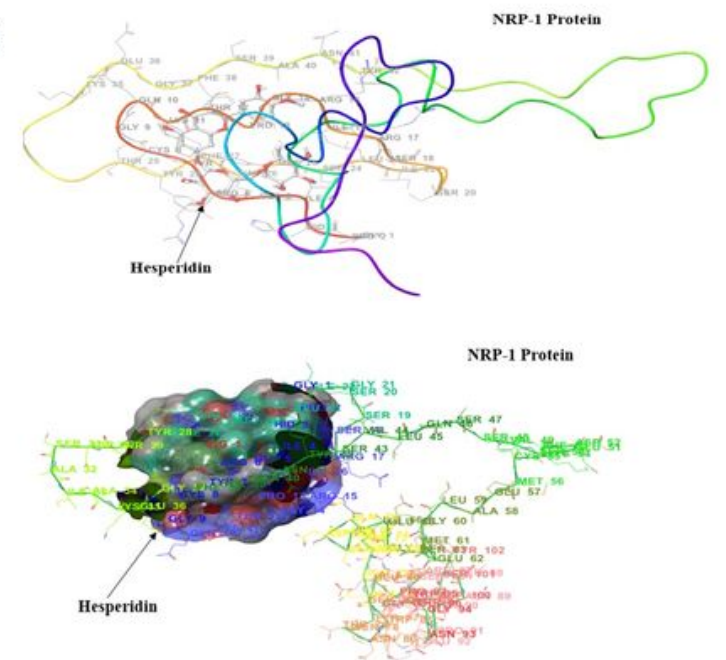

C
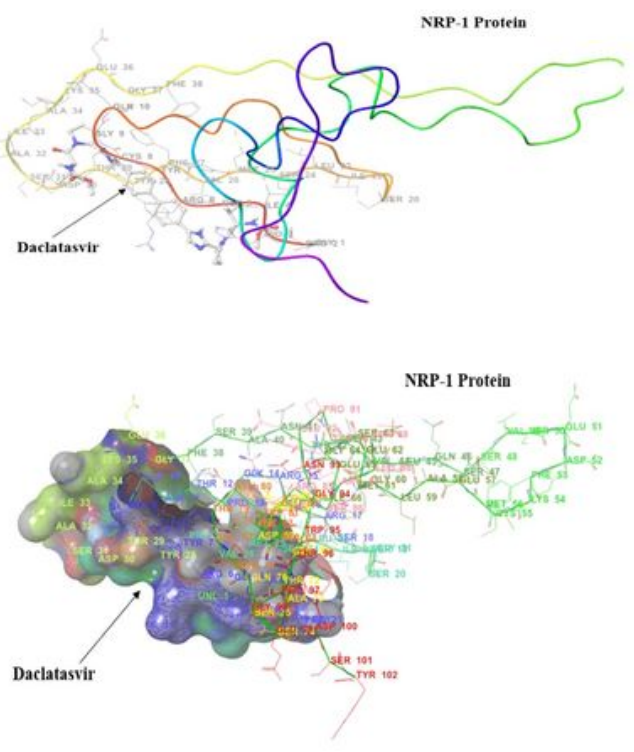

D
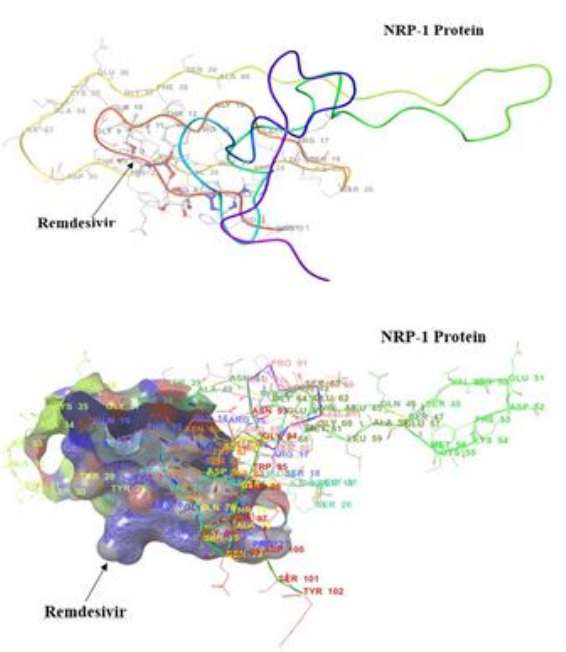

E
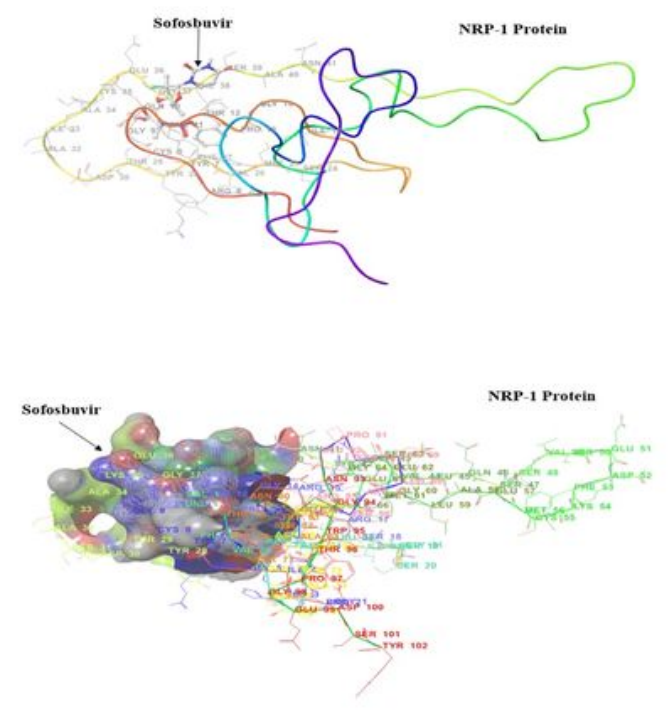

\section{Figure 2}

The docking complexes formed after the docking of (A) Ravidasvir, (B)Hesperidin, (C) Daclatasvir, (D) Remdesivir, and (E) Sofosbuvir into the Neuropilin-1 human receptor active site. 
A
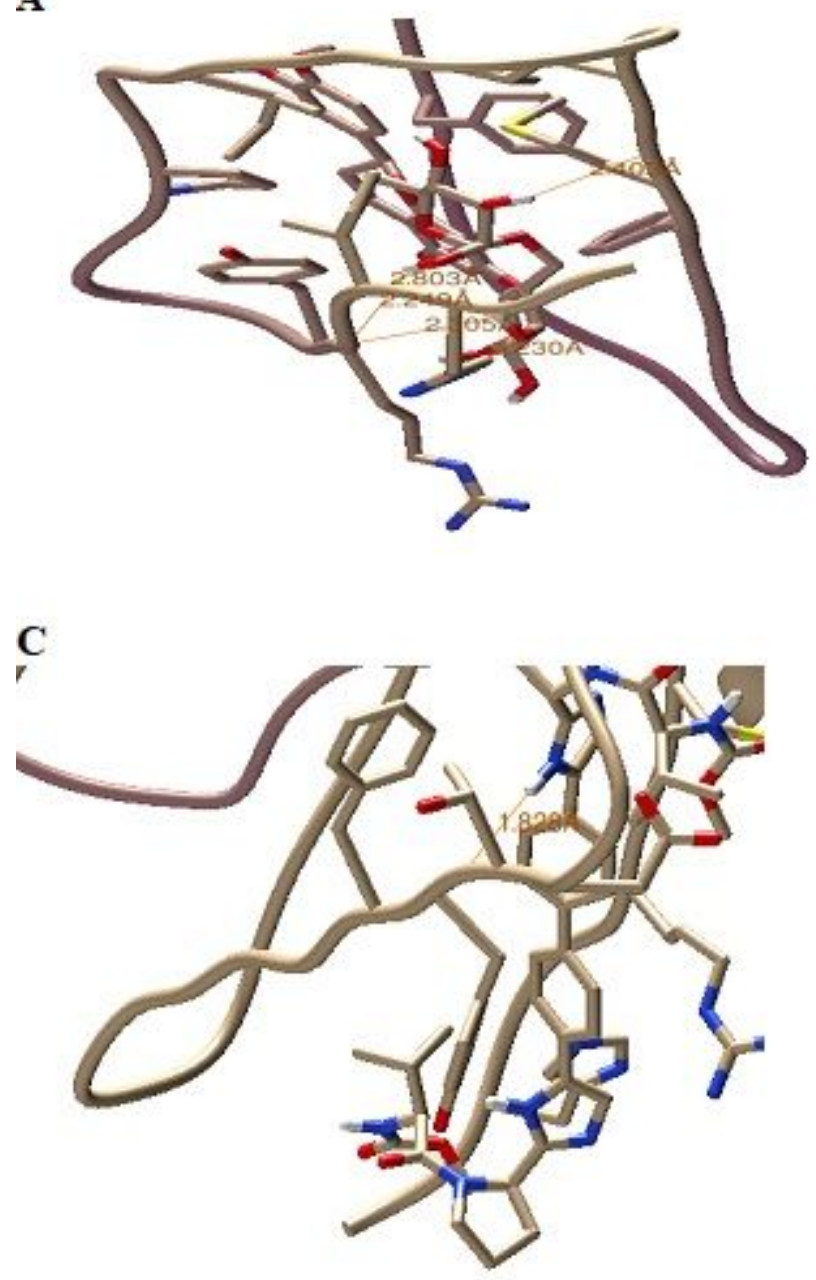

B

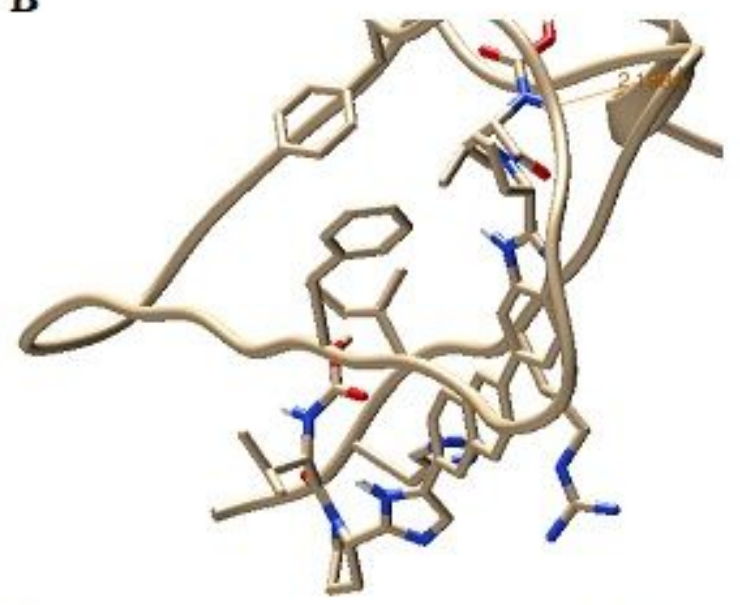

D

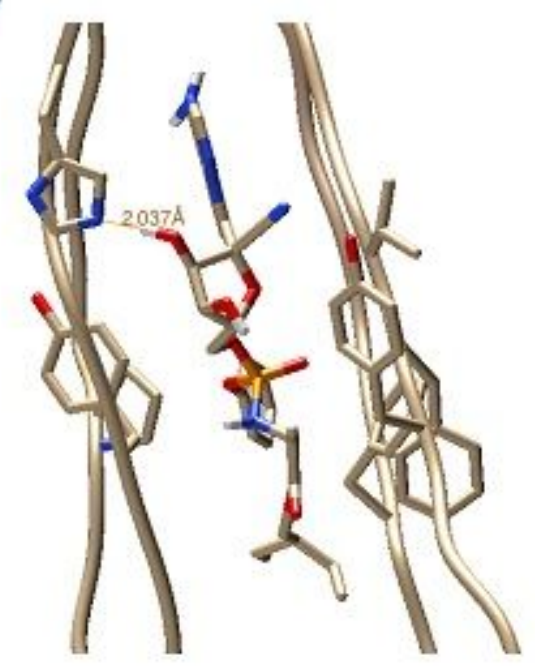

$\mathbf{E}$

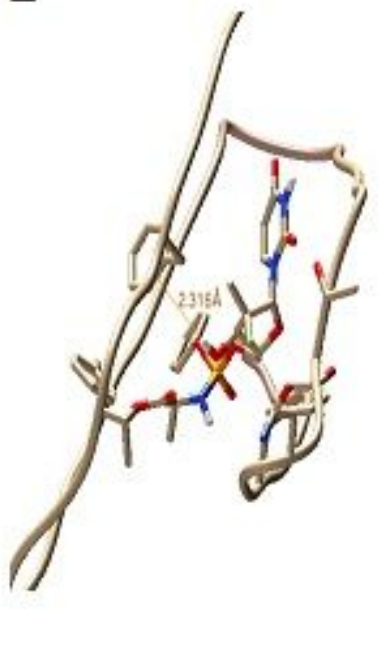

Figure 3

The Docked complexes of (A) Hesperidine, (B) Ravidasivir, (C) Daclatasvir, (D) Remdesivir and (E) Sofosbuvir with Neuropilin-1 model using Chimera software. 
A
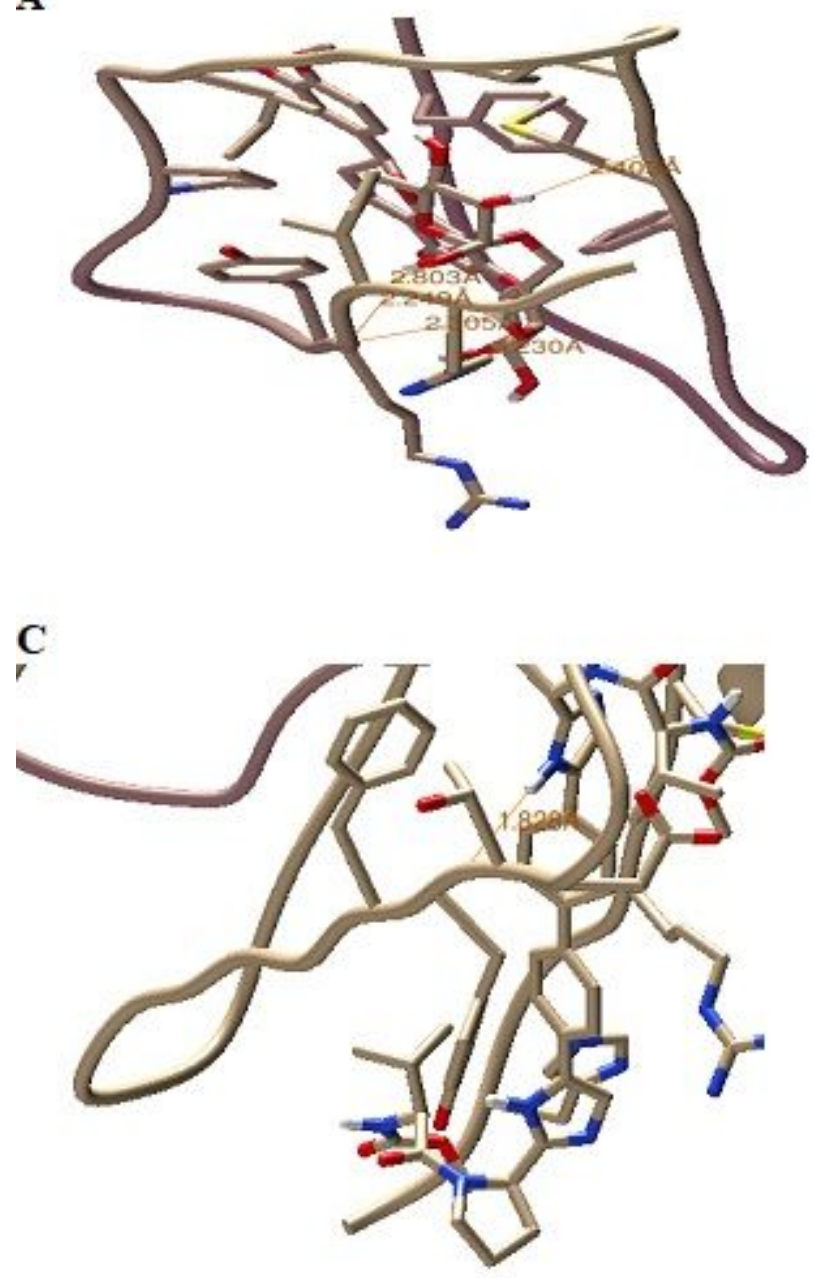

B

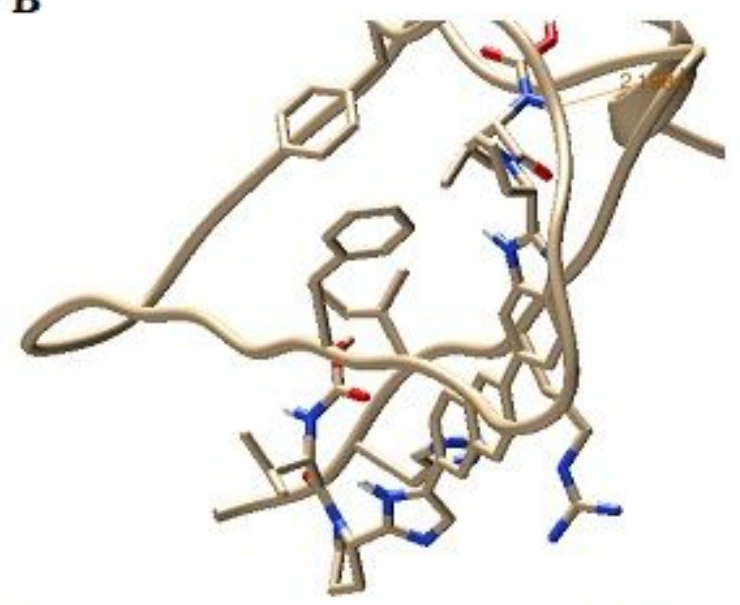

D

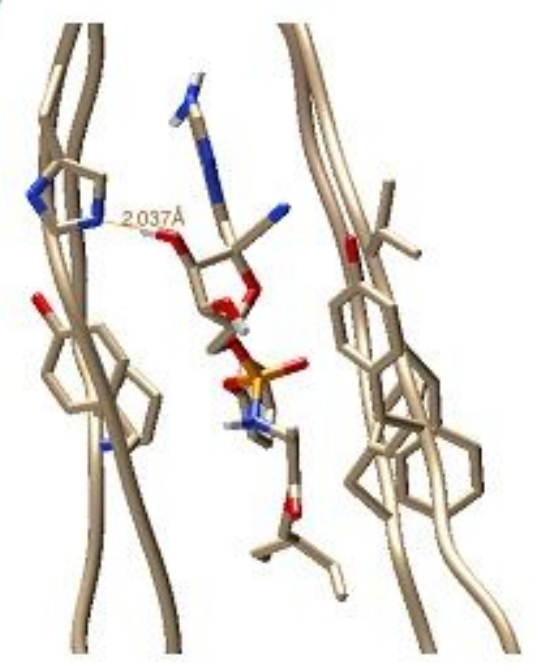

$\mathbf{E}$

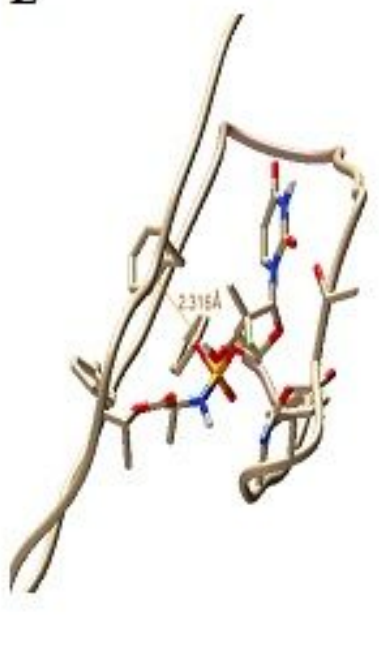

Figure 3

The Docked complexes of (A) Hesperidine, (B) Ravidasivir, (C) Daclatasvir, (D) Remdesivir and (E) Sofosbuvir with Neuropilin-1 model using Chimera software. 